\title{
The Study on the Application of Sensor Technology in the Development of Remote Monitoring System
}

\author{
Hou Wei ${ }^{1, a}$ \\ ${ }^{1}$ Chongqing College of Electronic Engineering, Chongqing, China, 401331 \\ a sina001007@163.com
}

Keywords: ZigBee; Wireless sensor network (WSN); Remote monitoring system; Development

\begin{abstract}
With the widely application of the ZigBee wireless sensor network, how to use the internet for real-time monitoring, control and management of remote monitoring area gradually become an important research topic in the field. ZigBee gateway in the wireless sensor network system plays an important role in hub, and it is also one of the "bottlenecks" of wireless sensor network as a whole system. The monitoring data of different monitoring area all over the world can achieve the purpose of sharing information through the gateway, which makes the physical world has smaller space and time distance, so the research that how to make the gateway transmission more efficient, real-time is very important. This article will mainly discuss the development and design for remote control system based on ZigBee wireless sensor network.
\end{abstract}

\section{Introduction}

Monitoring system is to help management of equipment and on-site real-time monitoring, prevention after the occurrence of failure or the failure to provide timely information. Automatic monitoring system can reduce the number of staff and improve the efficiency of monitoring business. Most of existing automatic monitoring system is using cable technology for sensor networks. Such schemes extension performance is poor, wiring lines, lines easy to aging, are also not convenient to replace. Build environment monitoring system using wireless way, you can avoid the unnecessary trouble. Moreover, relatively flexible wireless way, as long as within the effective range, sensor terminal can put according to the requirements. Wireless environment monitoring, however, has not been widely used, a major reason is that the current wireless communication product cost is very high, make its widely encountered stiff resistance. As humans in microelectronics mechanical systems in recent years, wireless communications, digital electronics has made great achievements, make the development of low cost, low power consumption, small volume, and short distance communication sensor possible. ZigBee will solve these problems; relative to other wireless network technology is more suitable for forming the environmental monitoring system.

\section{The architecture of wireless sensor network}

The realization of the wireless sensor network need ad-hoc network technology, compared with the general sense of self-organizing network, wireless sensor nodes in a network of numerous, overhead, it's for sensor network scalability requirements are put forward. Sensor networks typically do not have the world's only address identifier, which makes the sensor network of the network layer and transport layer relative to the general network has a lot of simplified. The greatest characteristic of self-organizing sensor network is the limited energy, limited by environmental sensor nodes, usually by the capacity is limited and cannot replace the batteries, so considering the sensor network system structure and each layer protocol design, energy-saving aspects of the design main consideration is. As a result of the sensor network application environment is special, sometimes damage probability than the traditional network sensor network node, and so you must guarantee the robustness of the self-organizing network, to ensure the damage of part of the sensor network does not affect the global task. Due to the high density of sensor nodes deployment, the characteristics of the network topology changes quickly, so the topology maintenance also puts 
forward the request. According to the above analysis, sensor networks need according to the different needs of users on the network, the design to adapt to the characteristics of network architecture, for the standardization of network protocols and algorithms provide a unified specification, enable it to meet the needs of users.

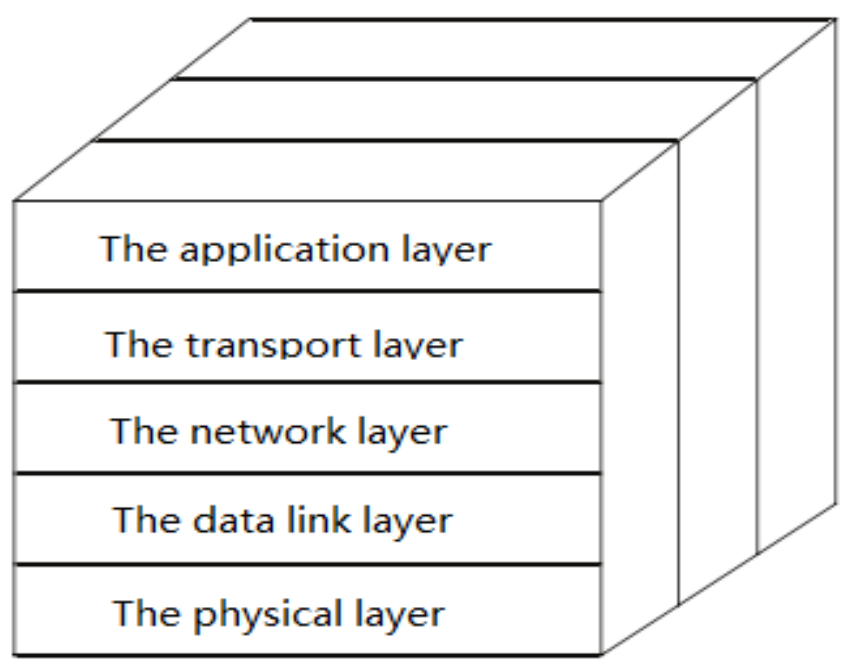

Figure 1 wireless sensor protocol stack

\section{The overview of ZigBee technology}

Wireless sensor network itself does not require higher transmission bandwidth, but need to lower power consumption and low transmission delay; this enables users with longer battery life and more array device. There is an urgent need to a low-end, control, application, simple and suitable for special standard of sensor networks. ZigBee has high communication efficiency, speed and low power consumption, low cost, high safety, and the advantages of fully digital. These advantages make the ZigBee and wireless sensor network (WSN) together perfectly. ZigBee is a new kind of wireless network technology, it is mainly used for wireless connectivity and networking from close range. It is based on the IEEE 802.15.4 standard, can realize mutual coordination communication between thousands of tiny sensors. ZigBee can use spectrum has three, respectively is $2.4 \mathrm{GHz}$ ISM band, 868 MHZ band of Europe, and America's 915 MHZ band, different frequencies available channel 1, 10, 16 respectively. ZigBee technology main application areas including industrial control, consumer electronics, automobile, home and building automation control, medical equipment, etc.

ZigBee is based on the IEEE 802.15.4 standard, and USES is with the same frequency is 2.4 $\mathrm{GHz} \mathrm{Wi}-\mathrm{Fi}$, it has 16 separate channels, that is to say, on a single location can provide 16 most network without interference. Within 100 meters maximum data transfer rate of up to $250 \mathrm{~KB} / \mathrm{s}$, compared with the Wi-Fi $54 \mathrm{MB} / \mathrm{s}$ or $1 \mathrm{MB} / \mathrm{s}$ of Bluetooth, the data rate is lower, can say this is ZigBee relative to the weakness of the other technologies. However, as previously mentioned, ZigBee is used for industrial purposes, in order to meet the demand of low power consumption and low cost is developed, and rate is not the key factor.

\section{The development and design of remote monitoring system}

Remote monitoring system USES the Browser/Server (B/S (Browser/Server) system model, so the requirement is a control system of the real-time monitoring and control system, the reliability, but also has the openness and universality of information system. As the comprehensive control system and information system, the emergence of remote monitoring system greatly facilitates the remote monitoring area of awareness and control.

The main function of design. Based on ZigBee wireless sensor network remote monitoring system to implement the main features are: using ZigBee wireless sensor network technology of 
wireless sensing monitoring area environment variable (in this topic for temperature, humidity); Through the gateway, and Web technology makes the remote monitoring center can be observed through the browser real-time monitoring area environment parameter, realize the sensor data storage, historical data query, monitoring parameter changes, such as abnormal automatic alarm function. Remote monitoring system design requirements and objectives are as follows:

a. The low cost, low power consumption. Sensor network node is made as a result of a AA battery power supply, so the requirements of small power consumption, sensor nodes can work long hours, to avoid the battery replacement often lead to additional waste of manpower and material resources.

b. The easy installation and maintenance. System adopts wireless communication network technology, avoid wiring, installation flexibility, system has a certain scale, according to the actual needs, flexible configuration monitoring network structure and the number of the nodes.

c. The sensor node is small in size, high precision. Sensor nodes can realize temperature and humidity parameter measurement, need has high measurement precision, wide range, strong anti-interference characteristics.

d. The software should be structured and modular program design, and as far as possible to do a single functional modularity. All the related modules are relatively independent and organic unity.

e. It can be controlled, safe. For different users have different access restrictions, administrators should have the highest authority, and general permissions users can only observe monitoring data. For orders issued, the user login, exit have detailed records, be safe and controllable.

$\mathrm{f}$. The monitoring center is fully functional, the client is easy to operate. The monitoring center can store a large number of sensor data, implementation, and at the same time, different observation, many people at the same time landing system; Browser client to real-time observation parameter data, historical data query, parametric curve display, networking of the sensor network.

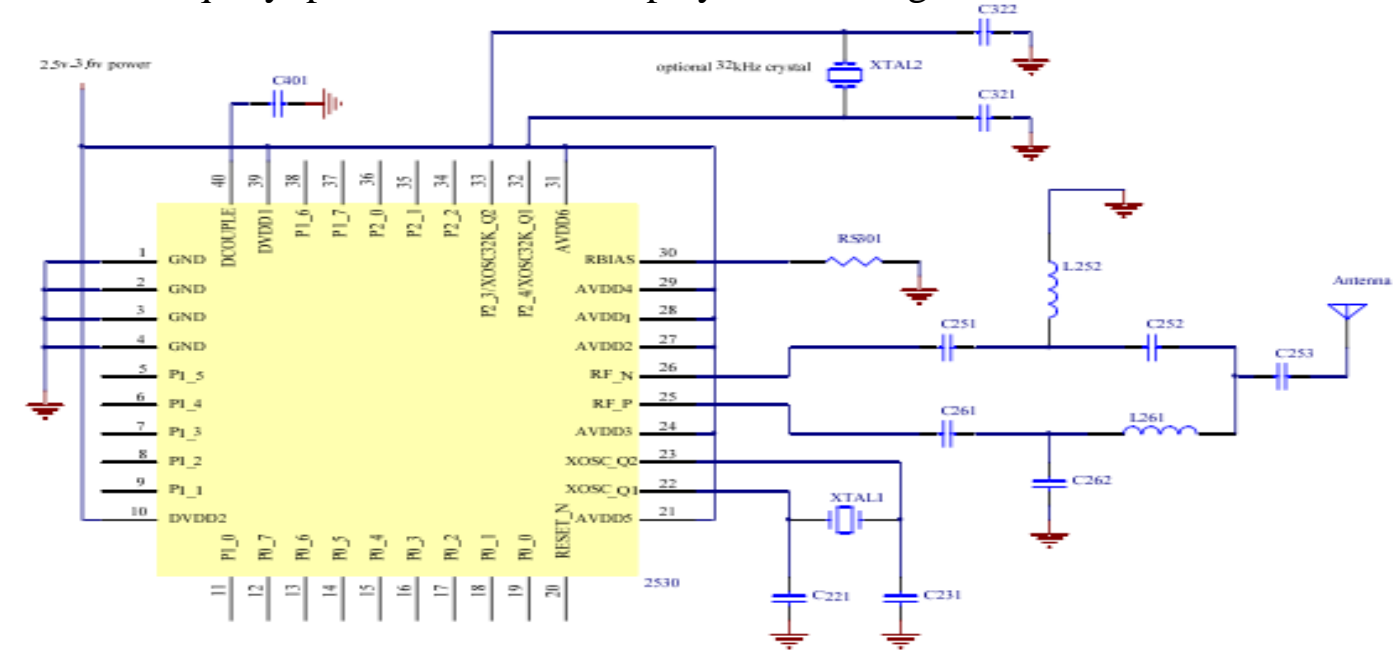

Fig. 2 CC2530 RF circuit diagram

ZigBee node hardware design. Wireless sensor nodes is a miniaturization of the embedded system, constitute the basis of wireless sensor network support platform. Different function of nodes needs different hardware. On the system hardware design, the most important thing is the design of the low power consumption; it is determined by the characteristics of wireless sensor network. In sensor network, sensor nodes and the fluid because of the location, required in the case of small size run as long as possible, so have high requirements for power consumption and stability. Low power design of system hardware mainly includes three aspects: simplify the hardware structure and low power consumption, small size of components selection.

This system uses the unbalanced monopole antenna; need to use a balance to optimize performance. Barron by low-cost discrete inductance and capacitance C262, L261 is implementation. 32 - MHz crystal vibration using an external 32 - MHz oscillator XTAL1 and two load capacitance (C221 and C231). XTAL2 is an optional $32.768 \mathrm{kHz}$ crystals, there are two load capacitance (C321 and C331) for $32.768 \mathrm{kHz}$ crystals. A very low $32.768 \mathrm{kHz}$ crystal is used for sleep current consumption and the application of precise time to wake up. 


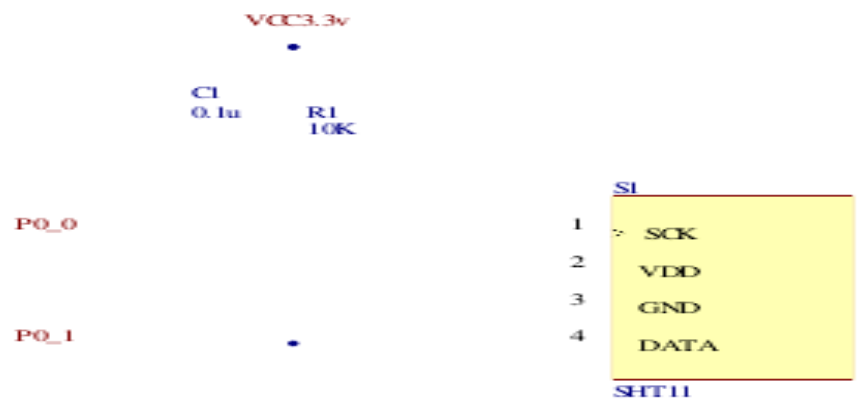

Figure 3 interface circuit principle diagram of temperature and humidity

From the function, the gateway is mainly responsible for data exchange between the two networks and control forwarding, gateway mainly consists of two logical entity: Ethernet control Process (E - Process, Ethernet Process), ZigBee network control Process (Z - Process, ZigBee Process) and the Initial Process (I - Process, Initial Process). E - the Process is mainly responsible for maintenance of Ethernet the interaction and network interface state, including opening, closing, listening socket and the socket of the reading and writing, etc.; Z - the Process is responsible for the ZigBee network interaction and network interface state, including the ZigBee network transmission network control command, receive from ZigBee Sink node ZigBee network packets, etc. However $\mathrm{E}$ - the Process need to $\mathrm{Z}$ - the Process of the sensor data is forwarded to the monitoring center, $\mathrm{Z}$ the Process need to E - Process receives commands via a serial port sent to the Sink node, so that two processes need a communication mechanism, we chose the first-in, first-out queue (FIFO, the First Input First Output) as inter-process communication mechanism, at the same time two processes need to share the equipment information table, so using the Shared memory. Initialization Process I - Process in Z - Process and E - Process run before the establishment of a complete device information table and communication FIFO.

\section{Summary}

Based on the existing wireless sensor network professional manufacturers to provide the solution of closed, difficulty in secondary development, the cost is expensive characteristic, the paper studies and designs a wireless sensor network based on ZigBee remote monitoring system, the system has the advantages of open, general and cost-effective use of the system can real-time monitor the environment parameters (such as temperature and humidity, etc.), real-time control monitoring area of the environment.

\section{References}

[1] Paolo Baronti,Prashant Pillai,Vince W.C. Chook,Stefano Chessa,Alberto Gotta,Y. Fun Hu. Wireless sensor networks: A survey on the state of the art and the 802.15.4 and ZigBee standards. Computer Communications. 2012 (7).

[2] Lilia Paradis,Qi Han. A Survey of Fault Management in Wireless Sensor Networks. Journal of Network and Systems Management. 2012 (2).

[3] Vivek Mhatre,Catherine Rosenberg. Design guidelines for wireless sensor networks: communication, clustering and aggregation. Ad Hoc Networks. 2013 (1).

[4] Jennifer Yick,Biswanath Mukherjee,Dipak Ghosal. Wireless sensor network survey. Computer Networks . 2012 (12).

[5] Ian F. Akyildiz,Tommaso Melodia,Kaushik R. Chowdhury. A survey on wireless multimedia sensor networks. Computer Networks . 2013 (4). 